\title{
Existence and Stability of Solutions to Non-Lipschitz Stochastic Differential Equations Driven by Lévy Noise
}

\author{
Yong $\mathrm{Xu}^{1)}$, Bin Pei \\ Department of Applied Mathematics, Northwestern Polytechnical University, Xi'an, \\ 710072, China
}

\begin{abstract}
In this paper, the successive approximation method is applied to investigate the existence and uniqueness of solutions to the stochastic differential equations (SDEs) driven by Lévy noise under non-Lipschitz condition which is a much weaker condition than Lipschiz one. The stability of the solutions to non-Lipschitz SDEs driven by Lévy noise is also considered, and the stochastic stability is obtained in the sense of mean square.
\end{abstract}

Keywords: Non-Lipschitz condition, Lévy noise, Existence and uniqueness, Successive approximation, Stability, Stochastic differential equations.

\section{Introduction}

In the real world, random fluctuations appear commonly in various natural and synthetic systems, and stochastic differential equations (SDEs) with random fluctuations and noises have been applied as the mathematical models of many practical systems. Such models can describe a great deal of different scientific and engineering problems, which appear in different fields including biology, epidemiology, mechanic, economics, finance and so forth [1-5]. So it is natural and necessary to investigate dynamical properties of the solutions to SDEs to find the effects of random perturbations in the corresponding realistic systems.

The mathematical models obtained have been greatly developed for SDEs under a random disturbance of the "Gaussian white noise", namely, the investigations

\footnotetext{
${ }^{1}$ Corresponding author. E-mail:hsux3@nwpu.edu.cn.
}

Tel./Fax. : 86-29 88431637 
concerning SDEs driven by Brownian motion have been very abundant up to now [4, 27]. However, Gaussian noises just like Brownian motion are not always appropriate while there exist large external and/or internal fluctuations with possible big jumps in some practical situations and environments. Unlike Brownian motion with the properties of no jump (the sample paths are continuous almost surely), normal diffusion (the mean square displacement increases linearly in time), and light tail or exponential relaxation (the probability density function decays exponentially in space) [4], non-Gaussian Lévy noise has completely peculiar properties of anomalous diffusion (mean square displacement is a nonlinear power law of time) [6] and heavy tail distribution or non-exponential relaxation [7]. Given the above-mentioned peculiar properties, non-Gaussian Lévy noise may be more appropriate to model the stochastic disturbances especially with extremely large jumps [8-11, 28-30]. For instance, recently, there has a growing interest in the use of Lévy process to model market behaviors in finance; not only are these of great mathematical interest but also there is growing evidence that they may be more realistic models than those that insist on continuous samples paths [12-14]. Therefore, it is significant to investigate the SDEs, properties of solutions and the applications with non-Gaussian Lévy noise.

A number of meritorious results concerning SDEs with non-Gaussian Lévy noise has been presented in existing literatures [7, 8, 15-17]. Among them, conditions which can guarantee the existence and uniqueness of the solutions to the SDEs with non-Gaussian Lévy noise are to be assumed as the one of the most basic and important Lipschitz condition. Generally, the Lipschitz case is a very common condition which has been widely used, and the existence and uniqueness of solutions to SDEs with non-Gaussian Lévy noise has been proved [16]. But, this condition is seemed to be considerably trenchant when one discusses variable applications in real world [7]. Thus, the importance to find some weaker and more generalized condition under which the SDEs with non-Gaussian Lévy noise still exist unique solutions is self-evident.

Fortunately, the so-called non-Lipschitz conditions have been proposed in [18-23], and by the method of the successive approximation, we in this paper prove the 
existence and uniqueness of solutions to SDEs driven by Lévy noise under this kind of non-Lipschitz condition with Lipschitz one as a special case. Furthermore, in order to obtain the dynamic properties of the solutions to non-Lipschitz SDEs driven by Lévy noise, we present the stability conditions and the theorem that the solutions are stochastic stable in the sense of mean square.

\section{Some preparations}

\subsection{Lévy motions}

Definition 1. Let $L(t)$ be a stochastic process defined on a probability $\operatorname{space}(\Omega, \mathcal{F}, P)$, if

(i) $L(0)=0 \quad$ (a.s.),

(ii) $L(t)$ has independent and stationary increments,

(iii) $L(t)$ is stochastically continuous, i.e. for all $a>0$ and for all $s \geq 0$,

$$
\lim _{t \rightarrow s} P(|L(t)-L(s)|>a)=0
$$

then $L(t)$ is a Lévy process.

A scalar Lévy process or motion is characterized by a drift parameter $q \in R^{d}$, a covariance $d \times d$ matrix $A$ and a nonnegative Borel measure $v$ defined on $\left(R^{d}, \mathcal{B}\left(R^{d}\right)\right)$ and concentrated on $R^{d} \backslash\{0\}$, which satisfies

$$
\int_{R^{d} \backslash\{0\}}\left(x^{2} \wedge 1\right) v(d x)<\infty .
$$

This measure $v$ is the so called Lévy jump measure of the Lévy process $L(t)$, and $(A, v, q)$ is defined as the generating triplet of Lévy motion.

It is known to all that a scalar Lévy motion is completely determined by the Lévy-Khintchine formula $[8,16,24]$. Now, we introduce the Lévy-Itô decomposition theorem with no proof and we may refer to [16] for more details.

Proposition 1. If $L(t)$ is a Lévy process in $R^{d}$, there exists $b_{1} \in R^{d}$, a Brownian 
motion $B(t)$ and independent Poisson random measure $N$ on $R_{+} \times\left(R^{d}-\{0\}\right)$, such that for each $t \geq 0$,

$$
L(t)=b_{1} t+B(t)+\int_{|x|<c} x \tilde{N}(t, d x)+\int_{|x| \geq c} x N(t, d x)
$$

where $N(d t, d x)$ is Poisson random measure, $\tilde{N}(d t, d x)=N(d t, d x)-v(d x) d t$ is the compensated Poisson random measure for $L(t)$ and $b_{1}=E\left(L(1)-\int_{|x| \geq c} x N(1, d x)\right)$ with the parameter $c \in[0, \infty)$ a constant.

\subsection{SDEs driven by Lévy noise}

We concern the SDEs with Lévy noise on $R^{d}$ :

$$
d X(t)=f(t, X(t-)) d t+g(t, X(t-)) d L(t), t \in[0, T] .
$$

Using the Lévy-Itô decomposition (1), we can rewrite this as follows

$$
\begin{aligned}
d X(t) & =f_{1}(t, X(t-)) d t+g(t, X(t-)) d B(t)+\int_{|x|<c} g(t, X(t-)) x \tilde{N}(d t, d x) \\
& +\int_{|x| \geq c} g(t, X(t-)) x N(d t, d x)
\end{aligned}
$$

where $f_{1}=b_{1}+f$.

Hence Eq. (2) can be rewritten in the following more general form:

$$
\begin{aligned}
d X(t) & =b(t, X(t-)) d t+\sigma(t, X(t-)) d B(t)+\int_{|x|<c} F(t, X(t-), x) \tilde{N}(d t, d x) \\
& +\int_{|x| \geq c} G(t, X(t-), x) N(d t, d x) .
\end{aligned}
$$

The third and fourth terms in the right hand side of Eq. (4) represent small and large jumps, and the term involving $G$ (or $F$ respectively) is absent when we take $c=\infty$. The term in Eq. (4) involving large jumps is controlled by $G$, which can be neglected through the technique of interlacing [16]. So we only focus on the study of an equation driven by continuous noise interspersed with small jumps, and then Eq. (4) can be modified as

$$
d X(t)=b(t, X(t-)) d t+\sigma(t, X(t-)) d B(t)+\int_{|x|<c} F(t, X(t-), x) \tilde{N}(d t, d x) .
$$

Then the integral form of Eq. (5) can be given 


$$
\begin{aligned}
X(t) & =X(0)+\int_{0}^{t} b(s, X(s-)) d s+\int_{0}^{t} \sigma(s, X(s-)) d B(s) \\
& +\int_{0}^{t} \int_{|x|<c} F(s, X(s-), x) \tilde{N}(d s, d x),
\end{aligned}
$$

with initial value $X(0)=\xi$, where $\xi$ is a given $d$-dimensional random vector. $b(t, \bullet)$ and $F(t, \bullet, x)$ are given $d$-dimensional random vector functions. $\sigma(t, \bullet)$ is a $d \times r$ matrix, $c$ is a positive constant, and $t \in[0, T], 0<T<+\infty . B(t)$ represents $r$-dimensional Brownian motion. We define the components of these terms as follows. For each $i$ and $j$, the mappings $b^{i}:[0, T] \times R^{d} \rightarrow R, \quad \sigma_{j}^{i}:[0, T] \times R^{d} \rightarrow R$ and $F^{i}:[0, T] \times R^{d} \times R^{d} \rightarrow R$ are all assumed to be measurable for $1 \leq i \leq d, 1 \leq j \leq r$.

\subsection{Non-Lipschitz condition}

Now, consider the following assumption on the coefficients of Eq. (6):

Assumption1. For each fixed $t \in[0, T]$, let $b(t, y), \sigma(t, y)$ and $F(t, y, x)$ be continuous in $y$, and $\forall y_{1}, y_{2} \in R^{n}$,

$$
\begin{aligned}
& \left|b\left(t, y_{1}\right)-b\left(t, y_{2}\right)\right|^{2}+\left\|\sigma\left(t, y_{1}\right)-\sigma\left(t, y_{2}\right)\right\|^{2} \\
& +\int_{|x|<c \mid}\left|F\left(t, y_{1}, x\right)-F\left(t, y_{2}, x\right)\right|^{2} v(d x) \leq \lambda(t) \kappa\left(\left|y_{1}-y_{2}\right|^{2}\right),
\end{aligned}
$$

where

(1a) $\lambda(t):[0, \infty) \rightarrow R^{+}$is an integrable function, $\kappa(q)$ is monotone non-decreasing, continuous and $\kappa(q)$ or $\kappa(q)^{2} / q$ is a concave function with respect to $q$ for fixed $t \geq 0$ with $\kappa(0)=0$ such that

$$
\begin{gathered}
\int_{0+} \frac{1}{\kappa(q)} d q=\infty, \\
\left\|\sigma\left(t, y_{1}\right)-\sigma\left(t, y_{2}\right)\right\|^{2}=\sum_{i=1}^{d} \sum_{j=1}^{r}\left(\sigma_{j}^{i}\left(t, y_{1}\right)-\sigma_{j}^{i}\left(t, y_{2}\right)\right)^{2}, \\
\left|b\left(t, y_{1}\right)-b\left(t, y_{2}\right)\right|^{2}=\sum_{i=1}^{d}\left(b^{i}\left(t, y_{1}\right)-b^{i}\left(t, y_{2}\right)\right)^{2},
\end{gathered}
$$




$$
\left|F\left(t, y_{1}, x\right)-F\left(t, y_{2}, x\right)\right|^{2}=\sum_{i=1}^{d}\left(F^{i}\left(t, y_{1}, x\right)-F^{i}\left(t, y_{2}, x\right)\right)^{2}
$$

(1c) $\sigma(t, 0), b(t, 0), F(t, 0, x)$ are integrable.

The Assumption 1 is the so-called non-Lipschitz conditions. In particular, if $\kappa(q)=q, \lambda(t)=K$, then the Assumption 1 reduces to Lipschitz condition. In other words, non-Lipschitz condition is weaker than Lipschitz condition.

Remark 1. For fixed $t \geq 0$, if $\kappa(q)$ or $\kappa(q)^{2} / q$ is concave function with respect to $q$, and $\forall y_{1}, y_{2} \in R^{n}$, the following inequality

$$
\begin{aligned}
& E\left|b\left(t, y_{1}\right)-b\left(t, y_{2}\right)\right|^{2}+E\left\|\sigma\left(t, y_{1}\right)-\sigma\left(t, y_{2}\right)\right\|^{2} \\
& +E \int_{|x|<c}\left|F\left(t, y_{1}, x\right)-F\left(t, y_{2}, x\right)\right|^{2} v(d x) \leq \lambda(t) \kappa\left(E\left|y_{1}-y_{2}\right|^{2}\right),
\end{aligned}
$$

holds.

The proof is given in $[18,23]$.

\section{Existence and uniqueness of solutions to non-Lipschitz SDEs with Lévy noise}

We in this section consider the existence and uniqueness of solutions to SDEs driven by Lévy noise under the above-mentioned non-Lipschitz condition. Hereafter, we assume without losing generality that $1 \leq T<+\infty$, and $K_{1}, C_{i}, i=1,2 \cdots 6$ are all constants.

Define a sequence of stochastic process $\left\{X_{k}(t)\right\}_{k=1,2 \ldots}$ with $X_{0}(t) \equiv \xi$ a random variable and $E|\xi|^{2}<+\infty$ as follows:

$$
\begin{gathered}
X_{k}(t)=\xi+\int_{0}^{t} b\left(s, X_{k-1}(s-)\right) d s+\int_{0}^{t} \sigma\left(s, X_{k-1}(s-)\right) d B(s) \\
+\int_{0}^{t} \int_{|x|<c} F\left(s, X_{k-1}(s-), x\right) \tilde{N}(d s, d x), k=1,2 \cdots
\end{gathered}
$$

\subsection{Existence of solutions to SDEs}


Theorem 1. Suppose that $b(t, y), F(t, y, x)$ and $\sigma(t, y)$ satisfy Assumption 1 , then

$$
\lim _{n, i \rightarrow \infty} E\left(\sup _{0 \leq t \leq T}\left|X_{n}(t)-X_{i}(t)\right|^{2}\right)=0 .
$$

By Theorem 1, we say $\left\{X_{k}(\cdot)\right\}_{k=1,2 \ldots}$ is a Cauchy sequence with its limit $X(\bullet)$. Then letting $n \rightarrow \infty$ in (9), we finally obtain that the solutions to Eq.

(2) exist.

To prove the Theorem 1, several auxiliary assertions are required.

Lemma 1. (Doob's martingale inequality) If $(X(t), t \geq 0)$ is a positive submartingale, then for any $p>1, t>0$,

$$
E\left[\sup _{0 \leq s \leq t} X(s)^{p}\right] \leq\left(\frac{p}{p-1}\right)^{p} E\left(X(t)^{p}\right) .
$$

Refer to [16] to obtain the proof.

Lemma 2. Under Assumption 1, there exists a positive number $K_{1}>0$ such that $\forall(t, y) \in[0, T] \times R^{d}$,

$$
|b(t, y)|^{2}+\|\sigma(t, y)\|^{2}+\int_{|x|<c}|F(t, y, x)|^{2} v(d x) \leq K_{1}\left(1+|y|^{2}\right) .
$$

Proof: Since $\kappa(q)$ or $\kappa(q)^{2} / q$ is a concave and non-negative function, such that $\kappa(0)=0$, we can choose two positive constants $a>0$ and $b>0$, so that [18]

$$
\kappa(q) \leq a+b q, q \geq 0 .
$$

Then, using (7) to yields

$$
\begin{aligned}
& |b(t, y)|^{2}+|| \sigma(t, y) \|^{2}+\int_{|x|<c}|F(t, y, x)|^{2} v(d x) \\
& \leq 2|b(t, y)-b(t, 0)|^{2}+2|b(t, 0)|^{2}+2\|\sigma(t, y)-\sigma(t, 0)\|^{2}+2\|\sigma(t, 0)\|^{2} \\
& +2 \int_{|x|<c}|F(t, y, x)-F(t, 0, x)|^{2} v d x+2 \int_{|x|<c \mid}|F(t, 0, x)|^{2} v(d x) \\
& \leq 2\left(|b(t, y)-b(t, 0)|^{2}+\|\sigma(t, y)-\sigma(t, 0)\|^{2}+\int_{|x|<c}|F(t, y, x)-F(t, 0, x)|^{2} v(d x)\right)
\end{aligned}
$$




$$
\begin{aligned}
& +2|b(t, 0)|^{2}+2\|\sigma(t, 0)\|^{2}+2 \int_{|x|<c}|F(t, 0, x)|^{2} v(d x) \\
& \leq 2 \sup _{0 \leq t \leq T}\left(|b(t, 0)|^{2}+\|\sigma(t, 0)\|^{2}+\int_{|x|<c}|F(t, 0, x)|^{2} v(d x)\right)+2 \lambda(t) \kappa\left(|y|^{2}\right) \\
& \leq K_{1}\left(1+|y|^{2}\right),
\end{aligned}
$$

where

$$
K_{1}=\max _{0 \leq t \leq T}\left\{2 \sup _{0 \leq t \leq T}\left(|b(t, 0)|^{2}+\|\sigma(t, 0)\|^{2}+\int_{|x|<c}|F(t, 0, x)|^{2} v(d x)+\lambda(t) a\right), 2 b \lambda(t)\right\}<+\infty .
$$

This completes the proof of Lemma 2.

Lemma 3. Under Lemma 2, one can have

$$
E\left|X_{k}(t)\right|^{2} \leq C_{1}, t \in[0, T], k=1,2 \cdots
$$

Proof : Here, for $k=1,2 \cdots$, we shall show

$$
E\left|X_{k}(t)\right|^{2} \leq 4 E|\xi|^{2} \sum_{l=0}^{k} \frac{\left(4 K_{1} T\right)^{l}}{l !} t^{l}+\sum_{l=1}^{k} \frac{\left(4 K_{1} T\right)^{l}}{l !} t^{l}
$$

Firstly, for $k=1$, it is easy to verify that

$$
\begin{aligned}
& E\left|X_{1}(t)\right|^{2} \\
& =E\left|\xi+\int_{0}^{t} b\left(s, X_{0}(s-)\right) d s+\int_{0}^{t} \sigma\left(s, X_{0}(s-)\right) d B(s)+\int_{0}^{t} \int_{|x|<c} F\left(s, X_{0}(s-), x\right) \tilde{N}(d s, d x)\right|^{2} \\
& \leq 4 E|\xi|^{2}+4 T E \int_{0}^{t}\left(\left|b\left(s, X_{0}(s-)\right)\right|^{2}+\|\left.\sigma\left(s, X_{0}(s-)\right)\right|^{2}+\int_{|x|<c}\left|F\left(s, X_{0}(s-), x\right)\right|^{2} v(d x)\right) d s \\
& \leq 4 E|\xi|^{2}+4 K_{1} T \int_{0}^{t}\left(1+E\left|X_{0}(s-)\right|^{2}\right) d s \\
& \leq 4 E|\xi|^{2}+4 K_{1} T\left(1+E|\xi|^{2}\right) t .
\end{aligned}
$$

So, (13) holds for $k=1$.

Secondly, assume that (13) holds for $k$, then, by induction, we have for $k+1$,

$$
\begin{aligned}
& E\left|X_{k+1}(t)\right|^{2} \\
& =E\left|\xi+\int_{0}^{t} b\left(s, X_{k}(s-)\right) d s+\int_{0}^{t} \sigma\left(s, X_{k}(s-)\right) d B(s)+\int_{0}^{t} \int_{|x|<c} F\left(s, X_{k}(s-), x\right) \tilde{N}(d s, d x)\right|^{2} \\
& \leq 4 E|\xi|^{2}+4 T E \int_{0}^{t}\left(\left|b\left(s, X_{k}(s-)\right)\right|^{2}+\left\|\sigma\left(s, X_{k}(s-)\right)\right\|^{2}+\int_{|x|<c \mid}\left|F\left(s, X_{k}(s-), x\right)\right|^{2} v(d x)\right) d s
\end{aligned}
$$




$$
\begin{aligned}
& \leq 4 E|\xi|^{2}+4 K_{1} T \int_{0}^{t}\left(1+4 E|\xi|^{2} \sum_{l=0}^{k} \frac{\left(4 K_{1} T\right)^{l}}{l !} t^{l}+\sum_{l=1}^{k} \frac{\left(4 K_{1} T\right)^{l}}{l !} t^{l}\right) d s \\
& =4 E|\xi|^{2}+4 K_{1} T t+4 E|\xi|^{2} \sum_{l=1}^{k+1} \frac{\left(4 K_{1} T\right)^{l}}{l !} t^{l}+\sum_{l=2}^{k+1} \frac{\left(4 K_{1} T\right)^{l}}{l !} t^{l} \\
& =4 E|\xi|^{2} \sum_{l=0}^{k+1} \frac{\left(4 K_{1} T\right)^{l}}{l !} t^{l}+\sum_{l=1}^{k+1} \frac{\left(4 K_{1} T\right)^{l}}{l !} t^{l} .
\end{aligned}
$$

Therefore, (13) holds for all $k$. Now, from (13), we obtain

$$
E\left|X_{k}(t)\right|^{2} \leq 4\left(1+E|\xi|^{2}\right) \exp \left\{4 K_{1} T^{2}\right\} .
$$

This completes the proof of Lemma 3.

Lemma 4. Suppose that $b(t, y), F(t, y, x)$ and $\sigma(t, y)$ satisfy the Assumption 1 , then for $t \in[0, T], \quad n \geq 1, k \geq 1$,

$$
\begin{aligned}
& E\left(\sup _{0 \leq s \leq t}\left|X_{n+k}(s)-X_{n}(s)\right|^{2}\right) \\
& \leq C_{2} \int_{0}^{t} \kappa\left[E\left(\sup _{0 \leq s_{1} \leq s}\left|X_{n+k-1}\left(s_{1}-\right)-X_{n-1}\left(s_{1}-\right)\right|^{2}\right)\right] d s,
\end{aligned}
$$

and

$$
E\left(\sup _{0 \leq s \leq t}\left|X_{n+k}(s)-X_{n}(s)\right|^{2}\right) \leq C_{3} t
$$

Proof: By Lemmas 1, 2 and Remark 1, we obtain

$$
\begin{aligned}
& E\left(\sup _{0 \leq s \leq t}\left|X_{n+k}(s)-X_{n}(s)\right|^{2}\right) \\
& \leq 3 E\left\{\sup _{0 \leq s \leq t}\left|\int_{0}^{s}\left[b\left(s_{1}, X_{n+k-1}\left(s_{1}-\right)\right)-b\left(s_{1}, X_{n-1}\left(s_{1}-\right)\right)\right] d s_{1}\right|^{2}\right\} \\
& +3 E\left\{\sup _{0 \leq s \leq t}\left|\int_{0}^{s}\left[\sigma\left(s_{1}, X_{n+k-1}\left(s_{1}-\right)\right)-\sigma\left(s_{1}, X_{n-1}\left(s_{1}-\right)\right)\right] d B\left(s_{1}\right)\right|^{2}\right\} \\
& +3 E\left\{\sup _{0 \leq s \leq t}\left|\int_{0}^{s} \int_{|x|<c}\left[F\left(s_{1}, X_{n+k-1}\left(s_{1}-\right), x\right)-F\left(s_{1}, X_{n-1}\left(s_{1}-\right), x\right)\right] \tilde{N}\left(d s_{1}, d x\right)\right|^{2}\right\}
\end{aligned}
$$




$$
\begin{aligned}
& \leq 3 T E \int_{0}^{t}\left|b\left(s, X_{n+k-1}(s-)\right)-b\left(s, X_{n-1}(s-)\right)\right|^{2} d s \\
& +12 E \int_{0}^{t}\left\|\sigma\left(s, X_{n+k-1}(s-)\right)-\sigma\left(s, X_{n-1}(s-)\right)\right\|^{2} d s \\
& +12 E \int_{0}^{t} \int_{|x|<c}\left|F\left(s, X_{n+k-1}(s-), x\right)-F\left(s, X_{n-1}(s-), x\right)\right|^{2} v(d x) d s \\
& \leq 12 T\left(\sup _{0 \leq s \leq T} \lambda(s)\right) \int_{0}^{t} \kappa\left[E\left(\sup _{0 \leq s_{1} \leq s}\left|X_{n+k-1}\left(s_{1}-\right)-X_{n-1}\left(s_{1}-\right)\right|^{2}\right)\right] d s .
\end{aligned}
$$

So, set $C_{2}=12 T\left(\sup _{0 \leq s \leq T} \lambda(s)\right)$, the inequality (15) holds.

Next, from Lemma 2, 3,

$$
\begin{aligned}
E\left(\sup _{0 \leq s \leq t}\left|X_{n+k}(s)-X_{n}(s)\right|^{2}\right) & \leq C_{2} \int_{0}^{t} \kappa\left(E\left(\sup _{0 \leq s_{1} \leq s}\left|X_{n+k-1}\left(s_{1}\right)-X_{n-1}\left(s_{1}\right)\right|^{2}\right)\right) d s \\
& \leq C_{2} \int_{0}^{t} \kappa\left(4 K_{1}\right) d s .
\end{aligned}
$$

Thus

$$
E\left(\sup _{0 \leq s \leq t}\left|X_{n+k}(s)-X_{n}(s)\right|^{2}\right) \leq C_{3} t
$$

This completes the proof of Lemma 4.

Now, we choose $0<T_{1} \leq T, t \in\left[0, T_{1}\right]$, such that

$$
\kappa_{1}\left(C_{3} t\right) \leq C_{3},
$$

where

$$
\kappa_{1}(q)=C_{2} \kappa(q)
$$

Then, for fixed $k \geq 1$, we introduce two sequences of functions $\left\{\phi_{n}(t)\right\}_{n=1,2 \ldots}$ and $\left\{\tilde{\phi}_{n, k}(t)\right\}_{n=1,2 \ldots}$, where

$$
\begin{gathered}
\phi_{1}(t)=C_{3} t \\
\phi_{n+1}(t)=\int_{0}^{t} \kappa_{1}\left(\phi_{n}(s)\right) d s \\
\tilde{\phi}_{n, k}(t)=E\left[\sup _{0 \leq s \leq t}\left|X_{n+k}(s)-X_{n}(s)\right|^{2}\right], n=1,2 \ldots
\end{gathered}
$$


Lemma 5. In terms of Assumption 1,

$$
0 \leq \tilde{\phi}_{n, k}(t) \leq \phi_{n}(t) \leq \phi_{n-1}(t) \leq \cdots \leq \phi_{1}(t), t \in\left[0, T_{1}\right]
$$

holds for each $k \geq 1$ and all positive integer $n \geq 1$.

Proof : Firstly, we shall show (18) for $n=1$. By Lemma 4, we have

$$
\tilde{\phi}_{1, k}(t)=E\left(\sup _{0 \leq s \leq t}\left|X_{1+k}(s)-X_{1}(s)\right|^{2}\right) \leq C_{3} t=\phi_{1}(t), t \in\left[0, T_{1}\right] .
$$

Secondly, it is easy to verify

$$
\begin{aligned}
\tilde{\phi}_{2, k}(t) & =E\left(\sup _{0 \leq s \leq t}\left|X_{2+k}(s)-X_{2}(s)\right|^{2}\right) \\
& \leq C_{2} \int_{0}^{t} \kappa\left[E\left(\sup _{0 \leq s_{1} \leq s}\left|X_{1+k}\left(s_{1}-\right)-X_{1}\left(s_{1}-\right)\right|^{2}\right)\right] d s \\
& \leq \int_{0}^{t} \kappa_{1}\left(\tilde{\phi}_{1, k}(s)\right) d s \leq \int_{0}^{t} \kappa_{1}\left(\phi_{1}(s)\right) d s=\phi_{2}(t) \\
& \leq \int_{0}^{t} \kappa_{1}\left(C_{3} s\right) d s \leq C_{3} t=\phi_{1}(t),
\end{aligned}
$$

thus, for $n=2$,

$$
\tilde{\phi}_{2, k}(t) \leq \phi_{2}(t) \leq \phi_{1}(t), t \in\left[0, T_{1}\right] .
$$

At last, by what assumed for $n$, for $n+1$, it is easy to verify

$$
\begin{aligned}
\tilde{\phi}_{n+1, k}(t) & =E\left(\sup _{0 \leq s \leq t}\left|X_{n+k+1}(s)-X_{n+1}(s)\right|^{2}\right) \\
& \leq C_{2} \int_{0}^{t} \kappa\left[E\left(\sup _{0 \leq s_{1} \leq s}\left|X_{n+k}\left(s_{1}-\right)-X_{n}\left(s_{1}-\right)\right|^{2}\right)\right] d s \\
& \leq \int_{0}^{t} \kappa_{1}\left(\tilde{\phi}_{n, k}(s)\right) d s \leq \int_{0}^{t} \kappa_{1}\left(\phi_{n}(s)\right) d s=\phi_{n+1}(t) \\
& \leq \int_{0}^{t} \kappa_{1}\left(\phi_{n-1}(s)\right) d s=\phi_{n}(t) .
\end{aligned}
$$

This completes the proof of Lemma 5.

\section{The proof of Theorem 1:}

Step 1: Since $\phi_{n}(t)$ decreases monotonically when $n \rightarrow \infty$ and $\phi_{n}(t)$ is non-negative function on $t \in\left[0, T_{1}\right]$, according to Lemma 5 , we can define the function $\phi(t)$ by $\phi_{n}(t) \downarrow \phi(t)$. Obviously, $\phi(0)=0$ and $\phi(t)$ is continuous function on $\left[0, T_{1}\right]$. Using the definition of $\phi_{n}(t)$ and $\phi(t)$, we reach 


$$
\phi(t)=\lim _{k \rightarrow \infty} \phi_{n+1}(t)=\lim _{k \rightarrow \infty} \int_{0}^{t} \kappa_{1}\left(\phi_{n}(s)\right) d s=\int_{0}^{t} \kappa_{1}(\phi(s)) d s, t \in\left[0, T_{1}\right] .
$$

Then, (19) implies $\phi(t) \equiv 0, t \in\left[0, T_{1}\right]$, since $\phi(0)=0$ and

$$
\int_{0+} \frac{d q}{\kappa_{1}(q)}=\frac{1}{C_{2}} \int_{0+} \frac{d q}{\kappa(q)}=+\infty
$$

Therefore we obtain

$$
0 \leq \lim _{k, n \rightarrow \infty} E\left(\sup _{0 \leq t \leq T_{1}}\left|X_{n+k}(t)-X_{n}(t)\right|^{2}\right)=\lim _{k, n \rightarrow \infty} \tilde{\phi}_{n, k}\left(T_{1}\right) \leq \lim _{n \rightarrow \infty} \phi_{n}\left(T_{1}\right)=0,
$$

namely,

$$
\lim _{n, i \rightarrow \infty} E\left(\sup _{0 \leq t \leq T_{1}}\left|X_{n}(t)-X_{i}(t)\right|^{2}\right)=0 .
$$

This completes the Step 1.

Step 2: Define

$$
T_{2}=\sup \left\{\tilde{T}: \tilde{T} \in[0, T] \text { and } \lim _{n, i \rightarrow \infty} E\left(\sup _{0 \leq t \leq \tilde{T}}\left|X_{n}(t)-X_{i}(t)\right|^{2}\right)=0\right\} .
$$

Immediately, we get $0<T_{1} \leq T_{2} \leq T$. Let $\varepsilon>0$ be an arbitrary positive number.

Choose $\delta$ so that $0<\delta<\min \left(T_{2}, 1\right)$ and

$$
C_{4} \delta<\frac{\varepsilon}{10}
$$

On one hand, from the definition of $T_{2}$, we have

$$
\lim _{n, i \rightarrow \infty} E\left(\sup _{0 \leq t \leq T_{2}-\delta}\left|X_{n}(t)-X_{i}(t)\right|^{2}\right)=0
$$

Thus, for large enough $N$, we observe

$$
E\left(\sup _{0 \leq t \leq T_{2}-\delta}\left|X_{n}(t)-X_{i}(t)\right|^{2}\right)<\frac{\varepsilon}{10}, n, i \geq N
$$

On the other hand, we obtain

$$
E\left(\sup _{T_{2}-\delta \leq t \leq T_{2}}\left|X_{n}(t)-X_{i}(t)\right|^{2}\right) \leq 3 I_{1}+3 I_{2}+3 I_{3},
$$

where 


$$
\begin{aligned}
& I_{1}=E\left(\sup _{T_{2}-\delta \leq t \leq T_{2}}\left|X_{n}(t)-X_{n}\left(T_{2}-\delta\right)\right|^{2}\right), \\
& I_{2}=E\left(\left|X_{n}\left(T_{2}-\delta\right)-X_{i}\left(T_{2}-\delta\right)\right|^{2}\right), \\
& I_{3}=E\left(\sup _{T_{2}-\delta \leq t \leq T_{2}}\left|X_{i}\left(T_{2}-\delta\right)-X_{i}(t)\right|^{2}\right) .
\end{aligned}
$$

Using Schwarz's inequality and Lemma1, we get

$$
\begin{aligned}
I_{1} & \leq 3 \delta E \int_{T_{2}-\delta}^{T_{2}}\left|b\left(s_{1}, X_{n-1}\left(s_{1}-\right)\right)\right|^{2} d s_{1}+12 E \int_{T_{2}-\delta}^{T_{2}}\left\|\sigma\left(s_{1}, X_{n-1}\left(s_{1}-\right)\right)\right\|^{2} d s_{1} \\
& +12 E \int_{T_{2}-\delta}^{T_{2}} \int_{|x|<c}\left|F\left(s_{1}, X_{n-1}\left(s_{1}-\right), x\right)\right|^{2} v(d x) d s_{1} \\
& \leq 12 \delta \int_{T_{2}-\delta}^{T_{2}} K_{1}\left(1+E\left|X_{n-1}\left(s_{1}-\right)\right|^{2}\right) d s_{1} \\
& \leq \delta C_{4},
\end{aligned}
$$

where $C_{4}=12 \delta K_{1}\left(1+C_{1}\right)$.

Therefore, by (21) we obtain

$$
I_{1} \leq \frac{\varepsilon}{10}
$$

and

$$
I_{3} \leq \frac{\varepsilon}{10}
$$

In addition, (22) implies

$$
I_{2}=E\left|X_{k}\left(T_{2}-\delta\right)-X\left(T_{2}-\delta\right)\right|^{2}<\frac{\varepsilon}{10}, k \geq N .
$$

Now, it is easy to verify

$$
\begin{aligned}
& E\left(\sup _{0 \leq t \leq T_{2}}\left|X_{n}(t)-X_{i}(t)\right|^{2}\right) \\
& \leq E\left(\sup _{0 \leq t \leq T_{2}-\delta}\left|X_{n}(t)-X_{i}(t)\right|^{2}\right)+E\left(\sup _{T_{2}-\delta \leq t \leq T_{2}}\left|X_{n}(t)-X_{i}(t)\right|^{2}\right) \\
& \leq \frac{\varepsilon}{10}+3 I_{1}+3 I_{2}+3 I_{3}<\varepsilon
\end{aligned}
$$

namely

$$
\lim _{n, i \rightarrow \infty} E\left(\sup _{0 \leq t \leq T_{2}}\left|X_{n}(t)-X_{i}(t)\right|^{2}\right)=0 .
$$

This completes the Step 2. 
Step 3:In this step we shall use the method of reduction to absurdity to show $T_{2}=T$.

Firstly, we give two lemmas:

Lemma 6. Assume $T_{2}<T$ and choose a sequence of number $\left\{a_{i}\right\}_{1 \leq i<n}$ so that $a_{i} \downarrow 0(i \rightarrow+\infty)$,

$$
E\left[\sup _{0 \leq t \leq T_{2}}\left|X_{n}(t)-X_{i}(t)\right|^{2}\right] \leq a_{i}
$$

then, for $n>i \geq 1$ we have

$$
E\left[\sup _{T_{2} \leq s \leq T_{2}+t}\left|X_{n}(s)-X_{i}(s)\right|^{2}\right] \leq 4 a_{i-1}+C_{5} t, T_{2}+t \leq T .
$$

Proof: First

$$
\begin{aligned}
& E\left(\sup _{T_{2} \leq s \leq T_{2}+t}\left|X_{n}(s)-X_{i}(s)\right|^{2}\right) \leq 4 E\left|X_{n-1}\left(T_{2}\right)-X_{i-1}\left(T_{2}\right)\right|^{2} \\
& +4 E\left|\sup _{T_{2} \leq s \leq T_{2}+t} \int_{T_{2}}^{s}\left[b\left(s_{1}, X_{n-1}\left(s_{1}-\right)\right)-b\left(s_{1}, X_{i-1}\left(s_{1}-\right)\right)\right] d s_{1}\right|^{2} \\
& +4 E\left|\sup _{T_{2} \leq s \leq T_{2}+t} \int_{T_{2}}^{s}\left[\sigma\left(s_{1}, X_{n-1}\left(s_{1}-\right)\right)-\sigma\left(s_{1}, X_{i-1}\left(s_{1}-\right)\right)\right] d B\left(s_{1}\right)\right|^{2} \\
& +4 E\left|\sup _{T_{2} \leq s \leq T_{2}+t} \int_{T_{2}}^{s} \int_{|x|<c}\left[F\left(s_{1}, X_{n-1}\left(s_{1}-\right), x\right)-F\left(s_{1}, X_{i-1}\left(s_{1}-\right), x\right)\right] \tilde{N}\left(d s_{1}, d x\right)\right|^{2} \\
& \leq 4 a_{i-1}+4 T E \int_{T_{2}}^{T_{2}+t}\left|b\left(s_{1}, X_{n-1}\left(s_{1}-\right)\right)-b\left(s_{1}, X_{i-1}\left(s_{1}-\right)\right)\right|^{2} d s_{1} \\
& +16 E \int_{T_{2}}^{T_{2}+t}\left\|\sigma\left(s_{1}, X_{n-1}\left(s_{1}-\right)\right)-\sigma\left(s_{1}, X_{i-1}\left(s_{1}-\right)\right)\right\|^{2} d s_{1} \\
& +16 E \int_{T_{2}}^{T_{2}+t} \int_{|x|<c}\left|F\left(s_{1}, X_{n-1}\left(s_{1}-\right), x\right)-F\left(s_{1}, X_{i-1}\left(s_{1}-\right), x\right)\right|^{2} v(d x) d s_{1} \\
& \leq 4 a_{i-1}+16 T\left(\sup _{0 \leq s \leq T} \lambda(s)\right)_{T_{2}}^{T_{2}+t} \kappa\left[E\left(\sup _{T_{2} \leq s_{1} \leq s}\left|X_{n-1}\left(s_{1}-\right)-X_{i-1}\left(s_{1}-\right)\right|^{2}\right)\right] d s \\
& \leq 4 a_{i-1}+C_{6} \int_{T_{2}}^{T_{2}+t} \kappa\left[E\left(\sup _{T_{2} \leq s_{1} \leq s}\left|X_{n-1}\left(s_{1}-\right)-X_{i-1}\left(s_{1}-\right)\right|^{2}\right)\right] d s,
\end{aligned}
$$

then 


$$
\begin{aligned}
E\left(\sup _{T_{2} \leq s \leq T_{2}+t}\left|X_{n}(s)-X_{i}(s)\right|^{2}\right) & \leq 4 a_{i-1}+C_{6} \int_{T_{2}}^{T_{2}+t} \kappa\left[E\left(\sup _{T_{2} \leq s_{1} \leq s}\left|X_{n-1}\left(s_{1}-\right)-X_{i-1}\left(s_{1}-\right)\right|^{2}\right)\right] d s \\
& \leq 4 a_{i-1}+C_{6} \int_{T_{2}}^{T_{2}+t} \kappa\left(4 C_{1}\right) d s_{1} \\
& \leq 4 a_{i-1}+C_{5} t
\end{aligned}
$$

This completes the proof of Lemma 6.

Next, choose a positive number $0<\eta \leq T-T_{2}$ and a positive integer $p$ so that

$$
C_{6} \kappa\left(4 a_{p-1}+C_{5} t\right) \leq C_{5}, \kappa_{2}(u)=C_{6} \kappa(u), t \in[0, \eta]
$$

Introduce the sequence of functions $\left\{\psi_{k}(t)\right\}_{k=1,2 \ldots}$ and $\left\{\tilde{\psi}_{k, n}(t)\right\}_{k, n \geq 1}$, defined by

$$
\begin{gathered}
\psi_{1}(t)=4 a_{p}+C_{5} t \\
\psi_{k+1}(t)=4 a_{p+k}+\int_{0}^{t} \kappa_{2}\left(\psi_{k}(s)\right) d s \\
\tilde{\psi}_{k, n}(t)=E\left(\sup _{T_{2} \leq s \leq T_{2}+t}\left|X_{n+k}(s)-X_{p+k}(s)\right|^{2}\right)
\end{gathered}
$$

Lemma 7. By Assumption 1,

$$
\tilde{\psi}_{k, n}(t) \leq \psi_{k}(t) \leq \psi_{k-1}(t) \leq \cdots \leq \psi_{1}(t), t \in[0, \eta],
$$

holds for all positive integer $k$.

Proof: We use Assumption 1, Eq. (25) and Lemma 6 to show it for $k=1,2$,

$$
\begin{aligned}
\tilde{\psi}_{1, n}(t) & \leq 4 a_{p}+C_{6} \int_{T_{2}}^{T_{2}+t} \kappa\left[E\left(\sup _{T_{2} \leq s_{1} \leq s}\left|X_{n}\left(s_{1}-\right)-X_{p}\left(s_{1}-\right)\right|^{2}\right)\right] d s \\
& \leq 4 a_{p}+\int_{T_{2}}^{T_{2}+t} \kappa_{2}\left(4 a_{p-1}+C_{5} s_{1}\right) d s_{1}=\psi_{1}(t),
\end{aligned}
$$

and 


$$
\begin{aligned}
\tilde{\psi}_{2, n}(t) & \leq 4 a_{p+1}+C_{6} \int_{T_{2}}^{T_{2}+t} \kappa\left[E\left(\sup _{T_{2} \leq s_{1} \leq s}\left|X_{n}\left(s_{1}-\right)-X_{p}\left(s_{1}-\right)\right|^{2}\right)\right] d s \\
& \leq 4 a_{p+1}+\int_{T_{2}}^{T_{2}+t} \kappa_{2}\left(\tilde{\psi}_{1, n}(t)\right) d s_{1} \leq 4 a_{p+1}+\int_{T_{2}}^{T_{2}+t} \kappa_{2}\left(\psi_{1}\left(s_{1}\right)\right) d s_{1} \\
& =\psi_{2}(t) \leq 4 a_{p}+\int_{T_{2}}^{T_{2}+t} \kappa_{2}\left(4 a_{p-1}+C_{5} s_{1}\right) d s_{1} \\
& \leq 4 a_{p}+C_{5} t=\psi_{1}(t), t \in[0, \eta] .
\end{aligned}
$$

Then we have proved

$$
\tilde{\psi}_{2, n}(t) \leq \psi_{2}(t) \leq \psi_{1}(t)
$$

Now assume that the assertion holds for $k \geq 2$. Then, by analogous argument, one obtains

$$
\begin{aligned}
\tilde{\psi}_{k+1, n}(t) & \leq 4 a_{p+k}+C_{6} \int_{T_{2}}^{T_{2}+t} \kappa\left[E\left(\sup _{T_{2} \leq s_{1} \leq s}\left|X_{n+k}\left(s_{1}-\right)-X_{p+k}\left(s_{1}-\right)\right|^{2}\right)\right] d s \\
& \leq 4 a_{p+k}+\int_{T_{2}}^{T_{2}+t} \kappa_{2}\left(\tilde{\psi}_{k, n}(t)\right) d s_{1} \\
& \leq 4 a_{p+k}+\int_{T_{2}}^{T_{2}+t} \kappa_{2}\left(\psi_{k}\left(s_{1}\right)\right) d s_{1}=\psi_{k+1}(t) \\
& \leq 4 a_{p+k-1}+\int_{T_{2}}^{T_{2}+t} \kappa_{2}\left(\psi_{k-1}\left(s_{1}\right)\right) d s_{1} \\
& =\psi_{k}(t), t \in[0, \eta] .
\end{aligned}
$$

Therefore, we obtain (27) for all $k$.

This completes the proof of Lemma 7.

In terms of (27), we can define the function $\psi(t)$ by $\psi_{k}(t) \downarrow \psi(t),(k \rightarrow \infty)$ and yield that

$$
\psi(0)=\lim _{k \rightarrow \infty} \psi_{k+1}(0)=\lim _{k \rightarrow \infty} a_{p+k}=0
$$

Since $\psi(t)$ is a continuous function on $[0, \eta]$, by the definition of $\psi_{k+1}(t)$ and $\psi(t)$, we have

$$
\psi(t)=\lim _{k \rightarrow \infty} \psi_{k+1}(t)=\lim _{k \rightarrow \infty}\left\{4 a_{p+k}+\int_{0}^{t} \kappa_{2}\left(\psi_{k}(s)\right) d s\right\}=\int_{0}^{t} \kappa_{2}(\psi(s)) d s .
$$


Then by $\psi(0)=0$ and

$$
\int_{0+} \frac{d q}{\kappa_{2}(q)}=\frac{1}{C_{6}} \int_{0+} \frac{d q}{\kappa(q)}=+\infty
$$

(29) implies $\psi(t)=0, t \in[0, \eta]$.

Therefore, we obtain

$$
\begin{aligned}
\lim _{k \rightarrow \infty} \tilde{\psi}_{k, n}(t) & =\lim _{k \rightarrow \infty} E\left(\sup _{0 \leq t \leq T_{2}+\eta}\left|X_{n+k}(s)-X_{p+k}(s)\right|^{2}\right) \\
& \leq \lim _{k \rightarrow \infty} E\left(\sup _{0 \leq t \leq T_{2}}\left|X_{n+k}(s)-X_{p+k}(s)\right|^{2}\right) \\
& +\lim _{k \rightarrow \infty} E\left(\sup _{T_{2} \leq t \leq T_{2}+\eta}\left|X_{n+k}(s)-X_{p+k}(s)\right|^{2}\right) \\
& \leq \lim _{k \rightarrow \infty} \psi_{k}(\eta)=\psi(\eta)=0,
\end{aligned}
$$

namely

$$
\lim _{n, i \rightarrow \infty} E\left(\sup _{0 \leq t \leq T_{2}+\eta}\left|X_{n}(t)-X_{i}(t)\right|^{2}\right)=0 .
$$

But, this conclusion is contradictory to the definition of $T_{2}$.

In other words, we have already shown that

$$
\lim _{n, i \rightarrow \infty} E\left(\sup _{0 \leq t \leq T}\left|X_{n}(t)-X_{i}(t)\right|^{2}\right)=0 .
$$

The proof of the existence of solutions to Eq. (2) is complete.

\subsection{Uniqueness of solutions to SDEs}

Theorem 2. Let $X(t)$ and $\tilde{X}(t)$ be two solutions to Eq. (2) on the same probability space such that $X(0)=\tilde{X}(0)$, then, under Assumption 1, the pathwise uniqueness holds for Eq. (2), $t \in[0, T]$.

Proof: By Cauchy-Schwarz inequality and Doob's martingale inequality, we observe that 


$$
\begin{aligned}
& E\left(|X(t)-\tilde{X}(t)|^{2}\right) \\
& =E \mid \int_{0}^{t}[b(s, X(s))-b(s, \tilde{X}(s))] d s+\int_{0}^{t}[\sigma(s, X(s))-\sigma(s, \tilde{X}(s))] d B(s) \\
& +\left.\int_{0}^{t} \int_{|x|<c}[F(s, X(s-), x)-F(s, \tilde{X}(s-), x)] \tilde{N}(d s, d x)\right|^{2} \\
& \leq 3 T E \int_{0}^{t}|b(s, X(s-))-b(s, \tilde{X}(s-))|^{2} d s+3 \int_{0}^{t}\|\sigma(s, X(s-))-\sigma(s, \tilde{X}(s-))\| \|^{2} d s \\
& +3 \int_{0}^{t} \int_{|x|<c \mid}|F(s, X(s-), x)-F(s, \tilde{X}(s-), x)|^{2} v(d x) d s \\
& \leq 3 T E \int_{0}^{t}|b(s, X(s-))-b(s, \tilde{X}(s-))|^{2}+\|\sigma(s, X(s-))-\sigma(s, \tilde{X}(s-))\|^{2} \\
& +\int_{|x|<c}|F(s, X(s-), x)-F(s, \tilde{X}(s-), x)|^{2} v(d x) d s .
\end{aligned}
$$

Noticing the Assumption 1, we have

$$
E\left(|X(t)-\tilde{X}(t)|^{2}\right) \leq 3 T E \int_{0}^{t} \lambda(s) \kappa\left(|X(s)-\tilde{X}(s)|^{2}\right) d s
$$

Since $\kappa(q)$ or $\kappa(q)^{2} / q$ is concave function, the above inequality (31) yields

$$
E\left(|X(t)-\tilde{X}(t)|^{2}\right) \leq 3 T \int_{0}^{t} \lambda(s) \kappa\left(E|X(s)-\tilde{X}(s)|^{2}\right) d s .
$$

Then, noticing that $\lambda(t)$ an integrable function and $\int_{0+} \frac{d u}{\kappa(q)}=+\infty$, the above inequality, as is well know, implies

$$
E\left(|X(t)-\tilde{X}(t)|^{2}\right)=0, t \in[0, T] .
$$

Since $T$ is an arbitrary positive number, we obtain from this $X(t) \equiv \tilde{X}(t)$ for all $0 \leq t \leq T$.

The proof of the uniqueness of solutions of SDEs (2) is complete.

\section{Stability of solutions}

Definition 1. A solution $X^{\xi}(t)$ of Eq.(2) with intial value $X(0)=\xi$ is said to be stable in mean square if for all $\varepsilon>0$ there exists $\delta>0$ such that when $E|\xi-\eta|^{2}<\delta$, 


$$
E\left(\sup _{0 \leq s \leq t}\left|X^{\xi}(s)-X^{\eta}(s)\right|^{2}\right) \leq \varepsilon
$$

where $X^{\eta}(t)$ is another solution of Eq.(2) with intial value $X(0)=\eta$.

In order to obtain the stability of solutions, we give two lemmas without details proof.

Lemma 8. (Bihari inequality) Let $T>0$ and $u_{0} \geq 0, u(t), v(t)$ be continuous functions on $[0, T]$. Let $\kappa: R^{+} \rightarrow R^{+}$be a concave continuous and non-decreasing function such that $\kappa(q)>0$ for $q>0$. If

$$
u(t) \leq u_{0}+\int_{0}^{t} v(s) \kappa(u(s)) d s, \text { for all } t \in[0, T]
$$

then

$$
u(t) \leq G^{-1}\left(G\left(u_{0}\right)+\int_{0}^{t} v(s) d s\right)
$$

for all $t \in[0, T]$ such that

$$
G\left(u_{0}\right)+\int_{0}^{t} v(s) d s \in \operatorname{Dom}\left(G^{-1}\right)
$$

where $G(q)=\int_{1}^{q} \frac{1}{\kappa(s)} d s, q \geq 0$ and $G^{-1}$ is the inverse function of $G$.

Lemma 9. Let the assumptions of Lemma 8 hold and $v(t) \geq 0$ for $t \in[0, T]$. If for all $\varepsilon>0$, there exists $t_{1} \geq 0$ such that for $0 \leq u_{0}<\varepsilon$,

$$
\int_{t_{1}}^{T} v(t) d t \leq \int_{u_{0}}^{\varepsilon} \frac{1}{\kappa(s)} d s
$$

holds. Then for every $t \in\left[t_{1}, T\right]$, the estimate $u(t) \leq \varepsilon$ holds.

Refer to $[25,26]$ to obtain proofs.

Theorem 3. Let $X^{\xi}(t)$ and $X^{\eta}(t)$ be two solutions of the Eq.(2) with intial value $\xi$ and $\eta$, respectively. The Assumption 1 is satisfied; then the solution of the Eq.(2) is said to be stable in mean square.

\section{Proof:}




$$
\begin{aligned}
& E\left(\sup _{0 \leq s \leq t}\left|X^{\xi}(s)-X^{\eta}(s)\right|^{2}\right) \leq 4 E|\xi-\eta|^{2} \\
& +16 t E \int_{0}^{t}\left|b\left(s, X^{\xi}(s-)\right)-b\left(s, X^{\eta}(s-)\right)\right|^{2}+\left\|\sigma\left(s, X^{\xi}(s-)\right)-\sigma\left(s, X^{\eta}(s-)\right)\right\|^{2} \\
& +\int_{|x|<c}\left|F\left(s, X^{\xi}(s-), x\right)-F\left(s, X^{\eta}(s-), x\right)\right|^{2} v(d x) d s \\
& \leq 4 E|\xi-\eta|^{2}+16 t\left(\sup _{0 \leq s \leq t} \lambda(s)\right) \int_{0}^{t} \kappa\left(E\left|\sup _{0 \leq s_{1} \leq s} X^{\xi}\left(s_{1}\right)-X^{\eta}\left(s_{1}\right)\right|^{2}\right) d s .
\end{aligned}
$$

Let

$$
\kappa_{3}(q)=16 T\left(\sup _{0 \leq s \leq T} \lambda(s)\right) \kappa(q)
$$

Thus, $\kappa_{3}(q)$ is obvious a monotone non-decreasing, continuous and concave function such that $\kappa_{3}(0)=0$ and $\int_{0+} \frac{1}{\kappa_{3}(q)} d q=\infty$. So for any $\varepsilon>0, \varepsilon_{1}:=\frac{\varepsilon}{2}$, we have $\lim _{s \rightarrow 0} \int_{s}^{\varepsilon_{1}} \frac{1}{\kappa_{3}(q)} d q=\infty$. Thus, there is a positive constant $\delta<\varepsilon_{1}$ such that $\int_{\delta}^{\varepsilon_{1}} \frac{1}{\kappa_{3}(q)} d q \geq T$

From the Lemma 9, let $u_{0}=4 E|\xi-\eta|^{2}, v(t)=1, u(t)=E\left(\sup _{0 \leq s \leq t}\left|X^{\xi}(s)-X^{\eta}(s)\right|^{2}\right)$. when $u_{0} \leq \delta \leq \varepsilon_{1}$, we have

$$
\int_{u_{0}}^{\varepsilon_{1}} \frac{1}{\kappa_{3}(q)} d q \geq \int_{\delta}^{\varepsilon_{1}} \frac{1}{\kappa_{3}(q)} d q \geq T=\int_{0}^{T} v(t) d t
$$

So, for any $t \in[0, T]$, the estimate $u(t) \leq \varepsilon$ holds.

This completes the proof.

\section{Acknowledgments}

This work was supported by the NSF of China (Grant Nos. 11372247, 11102157) and Shaanxi Province, Program for NCET, and SRF for ROCS, SEM, and NPU Foundation for New Faculties and Research Area Project and Garduate Strating Seed. 


\section{References}

[1] T. C. Gard, Introduction to stochastic differential equation, Dekker, New York, 1988.

[2] L. Arnold, Stochastic differential equations, Theory and Applications, John Wiley and Sons, (1974).

[3] A. Friedman, Stochastic Differential Equations and Applications, Dover Publications, 2006.

[4] B. Øksendal, Stochastic Differential Equations, sixth ed., Springer, Berlin, Heidelberg, 2005.

[5] C. Bianca, Onset of nonlinearity in thermostatted active particles models for complex systems, Nonlinear Analysis: Real World Applications. 13 (2012) 2593-2608.

[6] J. P. Bouchaud, A. Georges, Anomalous diffusion in disordered media: Statistic mechanics, models and physical applications, Phys. Rep. 195 (1990)127-293.

[7] R. Situ, Theory of Stochastic Differential Equations with Jumps and Applications, Springer, 2005.

[8] K. I. Sato, Lévy Processes and Infinitely Divisible Distributions, Cambridge University Press, Cambridge, 1999.

[9] F. Yonezawa, Introduction to focused session on 'anomalous relaxation', J. Non-Cryst. Solids (1996) 503-506.

[10]M. P. Herrchen, Stochastic modeling of dispersive diffusion by non-Gaussian noise, Doctorial Thesis, Swiss Federal Inst. of Tech. Zurich, 2001.

[11]]M. F. Shlesinger, G. M. Zaslavsky, U. Frisch, Lévy flights and related topics in physics, in: Lecture Notes in Physics, vol. 450, Springer-Verlag, Berlin, 1995.

[12]T. Chan, Pricing contingent claims on stocks driven by Levy processes, Annals Appl. Prob. 9 (1999) 504-28.

[13]H. Geman, D. B. Madan, M. Yor, Time changes for Lévy processes, Math. Finance 11 (2001) 79-86.

[14]D. B. Madan, E. Seneta, The variance gamma (V. G.) model for share market returns, J. Business 63 (1990) 511-24. 
[15]Y. Xu, J. Duan, and W. Xu, An averaging principle for stochastic dynamical systems with Lévy noise, Physica D. 240 (2011) 1395-1401

[16]D. Applebaum, Lévy processes and stochastic calculus, 2nd, Cambridge University Press, Cambridge, UK, 2009.

[17]D. Applebaum and M. Siakalli, Stochastic stabilization of dynamical systems using Lévy noise, Stoch. Dyn. 10(4) (2010) 509-527.

[18]T. Taniguchi, Successive approximations to solutions of stochastic differential equations, J. Differential Equations. 96 (1992) 152-169.

[19]W. Mao and X. Mao, On the approximations of solutions to neutral SDEs with Markovian switching and jumps under non-Lipschitz conditions, Appl. Math. Comput. 230(1) (2014) 104-109.

[20]T. Yamada, On the successive approximation of solutions of stochastic differential equations. J. Math. Kyoto Univ. 21(3) (1981) 501-515.

[21]S. Albeverio, Z. Brzézniak and J. Wu, Existence of global solutions and invariant measures for stochastic differential equations driven by Poisson type noise with non-Lipschitz coefficients, J. Math. Anal. Appl. 371 (2010) 309-322.

[22]B. Xie, Stochastic differential equations with non-Lipschitz coefficients in Hilbert spaces, Stochastic Anal. Appl. 26(2) (2008) 408-433.

[23]L. Wang, T. Cheng and Q. Zhang, Successive approximation to solutions of stochastic differential equations with jumps in local non-Lipschitz conditions, Appl. Math. Comput. 225 (2013) 142-150.

[24]S. Peszat and J. Zabczyk, Stochastic partial differential equations with Lévy Noise: An Evolution Equation Approach, Cambridge University Press, Cambridge, 2007.

[25]Y. Ren and N. M. Xia, Existence, uniqueness and stability of the solutions to neutral stochastic functional differential equations with infinite delay, Appl. Math. Comput. 210(1) (2009) 72-79.

[26]I. Bihari, A generalization of a lemma of Bellman and its application to uniqueness problem of differential equations, Acta Math. Acad. Sci. Hungar. 7 (1956) 71-94. 
[27]X. R. Mao, Stochastic differential equations and applications, Harwood, New York, 1997.

[28]X. M. Liu, J. Q. Duan, J. C. Liu, et al. Synchronization of systems of Marcus canonical equations driven by $\alpha$-stable noises, Nonlinear Analysis: Real World Applications. 11(2010)3437-3445.

[29]Z. Yang, G. Yin. Stability of nonlinear regime-switching jump diffusion, Nonlinear Analysis: Theory, Methods \& Applications., 75(9) (2012) 3854-3873.

[30]G. Yin, H. Yang, Two-time-scale jump-diffusion models with Markovian switching regimes, Stochastics and Stochastic Reports. 76(2)(2004)77-99. 